\title{
DETERMINATION OF DIAGNOSTIC ACCURACY OF BIOCHEMICAL PARAMETERS (CRP, LDH \& FERRITIN) IN THE DIAGNOSIS OF COVID-19 IN SUSPECTED COVID CASES
}

\author{
Tayyaba Ashiq, Abdus Sattar*, Nasir Uddin, Qamar Bashir, Sajida Shaheen**, Aamir Ijaz ${ }^{* * *}$ \\ Combined Military Hospital Lahore/National University of Medical Sciences (NUMS) Pakistan, *Combined Military Hospital Lahore Medical College, \\ Lahore/National University of Medical Sciences (NUMS) Pakistan, ${ }^{* *}$ Combined Military Hospital Peshawar/National University of Medical Sciences (NUMS)

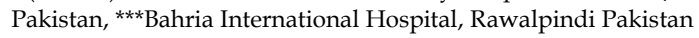

\begin{abstract}
Objective: To determine the diagnostic accuracy of the Lactate Dehydrogenase, C-Reactive Protein and Ferritin in suspected patients of COVID-19.

Study Design: Cross-sectional validation study.

Place and Duration of Study: Pathology department of Combined Military Hospital Lahore in the month of May 2020.

Methodology: We included 101 adult (>18 years) symptomatic suspected COVID-19 patients of both genders. Children, pregnant women and asymptomatic patients were excluded from study. Age, gender and results of Reverse Transcriptase Polymerase Chain Reaction, Lactate Dehydrogenase, C-Reactive Protein, ferritin were recorded.

Results: Lactate Dehydrodenase had highest sensitivity (75\%) with positive predictive value of $71.6 \%$ and diagnostic accuracy of $65.3 \%$ among three biochemical parameters studied. Receiver Operator Characteristic curve was studied. Area under curve of Lactate Dehydrogenase (AUC $=0.65)$ and Ferritin $(A U C=0.59)$ reflected their ability to prognosticate the presence of COVID19 disease. However, C-Reactive Protein $(\mathrm{AUC}=0.42)$ appeared to be a poor predictor of the disease.

Conclusion: Raised serum Lactate Dehydrogenase (>490 U/L) and Ferritin (>152 ng/L) levels can be used to predict the Reverse Transcriptase Polymerase Chain Reaction positivity for COVID-19 in the population of suspected patients of COVID19. However, C-Reactive Protein is a poor predictor of COVID-19.
\end{abstract}

Keywords: C-Reactive protein, COVID-19, Diagnostic accuracy, Ferritin, Lactate dehydrogenase, Reverse transcriptase polymerase chain reaction.

How to Cite This Article: Ashiq T, Sattar A, Uddin N, Bashir Q, Shaheen S, Ijaz A. Determination of Diagnostic Accuracy of Biochemical Parameters (CRP, LDH E FERRITIN) in The Diagnosis of COVID-19 in Suspected COVID Cases. Pak Armed Forces Med J 2021; 71(5): 1722-1726. doi: https://doi.org/10.51253/pafmj.v71i5.5749

This is an Open Access article distributed under the terms of the Creative Commons Attribution License (https://creativecommons.org/licenses/by-nc/4.0/), which permits unrestricted use, distribution, and reproduction in any medium, provided the original work is properly cited.

\section{INTRODUCTION}

The single stranded ribonucleic acid (RNA) virus of Corona virus family called Severe Acute Respiratory Syndrome-Corona virus-2 (SARS-CoV-2) is responsible for a respiratory illness called Corona Virus Disease-2019 (COVID-19)1,2. In December 2019, the outbreak of COVID-19 started from Wuhan city of China and subsequently spread to more than 150 countries across the globe. On 11 March 2020, World Health Organization (WHO) declared it a Pandemic. ${ }^{1}$

The first case of COVID-19 was confirmed in Pakistan on February 26 2020 from Karachi. ${ }^{2}$ Later, the virus spread to various regions of the country. According to report of COVID-19 health advisory platform Ministry of National Health Services, till 10 th April 2020, 54706 suspected corona virus patients were reported in Pakistan, $8.6 \%$ of which tested positive for COVID-19. ${ }^{3}$ The rate of infection COVID-19 virus was estimated to be 2.3 per 100,000 population of Pakistan.

Correspondence: Dr Tayyaba Ashiq, Resident Chemical Pathologist, Combined Military Hospital, Lahore Pakistan

Received: 27 Nov 2020; revision received: 17 May 2021; accepted: 19 May 2021
About $49 \%$ of patients were registered in the Punjab. ${ }^{4}$

The clinical spectrum of COVID-19 ranges from asymptomatic infection to severe pneumonia with respiratory failure and even death. ${ }^{2}$ Most of the patients remain asymptomatic despite being active carriers $^{5}$. Person to person transmission is believed to occur via respiratory droplets. ${ }^{2}$ Early identification of infection and timely isolation and management is crucial to control the disease. The real time Reverse transcriptase Polymerase Chain Reaction (RT-PCR) becomes positive within first week of exposure and is used for its diagnosis. ${ }^{6}$ Initial studies implicate vasculitic processes in pathogenesis of organ damage. These mechanisms are propagated by activation of complement system, pro-inflammatory and inflammatory cytokines. ${ }^{7}$ The disease is associated with a rise in certain biochemical parameters like C-Reactive Protein (CRP), Lactate Dehydrogenase (LDH) and Ferritin. ${ }^{7-9}$ CRP has been found to be prominently increased in initial phase of infection and is an early predictor of infection. It is also associated with disease progression.7,10 Ferritin has been reported to be markedly raised in nonsurvivor than survivors. ${ }^{7}$ Raised LDH indicates tissue 
destruction and is an important prognostic marker of lung injury.

Our study aims to determine the diagnostic accuracy and association of these biochemical parameters with COVID-19 in suspected cases using RT-PCR as a gold standard for diagnosis.

Whether these biochemical parameters can be used as surrogate markers of COVID-19 positivity is a dilemma. This can help in early isolation and management of suspected patients of COVID-19 because results of biochemical tests can be generated within 2-3 hours as compared to PCR which may take 12-24 hours. An important aspect of COVID-19 diagnosis is that molecular diagnostic facilities are expensive and are available only at selected centers. However, facility for analysis of biochemical parameters is easily available and can be used to screen the masses. The objective of our study was to determine diagnostic accuracy of LDH, CRP \& Ferritin in symptomatic suspected COVID-19 patients, using RT-PCR as a gold standard for its diagnosis.

\section{METHODOLOGY:}

This cross-sectional validation study was conducted in Pathology department of Combined Military Hospital (CMH) Lahore after taking approval from Research Review Board of the hospital (Ref no. 248/ 2020). The sample size was calculated using following equation (A1) taken from a study by Flahault et al on sample size calculation design accuracy in diagnostic test studies ${ }^{11}$.

Inclusion Criteria: The adults (age $>18$ years) suspected COVID-19 patients of both genders, reporting to the screening center for their symptoms (fever, cough, flu, diarrhea, difficulty in breathing) or positive travel/ contact history with COVID-19 patient, from May to May 2020, were included in the study, using nonprobability convenient sampling technique.

Exclusion Criteria: Children, pregnant women and asymptomatic patients were excluded from the study.

Informed consent was taken from them. On admission Throat swab for RT-PCR for COVID-19 virus was taken in viral transport medium (VTM). The blood samples $(3 \mathrm{ml})$ were taken in plain tubes for biochemical parameters. Serum was separated by centrifugation at $3500 \mathrm{rpm}$ for 3 minutes and was analyzed without any delay. Sera were kept at $2-8^{\circ} \mathrm{C}$, if there was any delay in analysis. Roche Cobas C-501 random access chemistry auto-analyzer was used for analysis of C-reactive protein (CRP) and lactate dehydrogenase (LDH). For Ferritin, Roche Cobas e-411 auto-analyzer was used.

Age, gender and result of RT-PCR along with biochemical parameters (LDH, CRP \& Ferritin) were recorded and entered in SPSS (version 21) for statistical analysis. Frequency of age gender and RT-PCR result was determined. Sensitivity, specificity, positive predictive values (PPV), negative predictive value (NPV) and diagnostic accuracy for each studied biochemical parameter were calculated. We used chi-square test to find the difference between frequency of patients with the low and raised levels of biochemical parameters in RT-PCR positive and negative cases. The Receiver Operator Characteristic (ROC) graph was made to see the area under the curve (AUC) of the studied parameters to prognosticate the patients with positive RT-PCR for COVID-19.

\section{RESULTS}

One hundred and one suspected patients of COVID-19 were enrolled in this study, $72.3 \%$ were males and $27.7 \%$ were females. Mean age of patients was $46.7 \pm 16.9$ years. Most of patients $(46.5 \%)$ were young, lying in the age range of 18 to 40 years. 33.7\% of cases were aged between $41-60$ years and only $19.8 \%$ were above 61 years of age. The result of RT-PCR for COVID-19 was positive in $64(63.4 \%)$ and negative in $37(36.6 \%)$ cases. Out of 64 COVID-19 cases, 48 had raised LDH levels (Table-I). Among the 37 negative cases, 13 and 6 had raised and severely raised LDH levels respectively (Table-II). Surprisingly 32 COVID19 positive cases had CRP level within normal reference limits. $17 \%, 25 \%$ and $10 \%$ of positive COVID-19

Table-I: Sensitivity, specificity, positive predictive value, negative predictive value and diagnostic accuracy of biochemical parameters.

\begin{tabular}{|c|c|c|c|}
\hline Diagnostic Parameters & $\begin{array}{c}\text { Lactate } \\
\text { Dehydrogenase }\end{array}$ & Ferritin & $\begin{array}{c}\text { C-Reactive } \\
\text { Protein }\end{array}$ \\
\hline Sensitivity $=$ True Positive /( True Positive +False Negative) & $75 \%$ & $54.6 \%$ & $50 \%$ \\
\hline Specificity $=$ True Negative /(True Negative + False Positive $)$ & $48 \%$ & $43.2 \%$ & $40.5 \%$ \\
\hline Positive Predictive Value= True Positive/(True Positive+ False Positive) & $71.6 \%$ & $62.5 \%$ & $59.3 \%$ \\
\hline Negative Predictive Value= True Negative/(True Negative +False Negative) & $52.9 \%$ & $35.5 \%$ & $31.9 \%$ \\
\hline Diagnostic Accuracy $=($ True Positive + True Negative $) /$ All Patients & $65.3 \%$ & $55.4 \%$ & $46.5 \%$ \\
\hline
\end{tabular}

LDH: Lactate Dehydrogenase, CRP: C-Reactive Protein. 
Diagnostic Accuracy of Biochemical Parameters in COVID

cases had high normal, moderately raised and markedly raised Ferritin levels respectively (Table-II). nation, 0.7-0.8 is acceptable, 0.8-0.9 is excellent, and $>0.9$ is considered outstanding.

Table-II: Results of biochemical parameters in COVID-19 patients.

\begin{tabular}{|c|c|c|c|c|c|}
\hline \multirow[t]{2}{*}{ Parameters } & \multirow[t]{2}{*}{ Values } & \multicolumn{3}{|c|}{$\begin{array}{l}\text { Reverse Transcriptase Polymerase Chain Reaction } \\
\text { (RT-PCR) }\end{array}$} & \multirow[t]{2}{*}{$p$-value } \\
\hline & & Positive & Negative & Total cases & \\
\hline \multirow{3}{*}{$\begin{array}{l}\text { Lactate } \\
\text { Dehydrogenase } \\
(\mathrm{LDH})\end{array}$} & 150-450 U/L (ref. limit) & $16(47)$ & $18(53)$ & 34 & \multirow{3}{*}{$0.02^{*}$} \\
\hline & 450-1000 U/L ( mildly raised) & $40(75)$ & $13(25)$ & 53 & \\
\hline & $>1000 \mathrm{U} / \mathrm{L}$ (markedly raised) & $8(57)$ & $6(43)$ & 14 & \\
\hline \multirow{4}{*}{ Ferritin } & $12-100$ ng/L (low normal) & $12(46)$ & $14(54)$ & 26 & \multirow{4}{*}{$0.22^{* *}$} \\
\hline & $100-200$ ng/L (high normal) & $17(71)$ & $7(29)$ & 24 & \\
\hline & 200-1000 ng/L ( mildly raised) & $25(67)$ & $12(33)$ & 37 & \\
\hline & $>1000 \mathrm{ng} / \mathrm{L}$ ( markedly raised) & $10(71)$ & $4(29)$ & 14 & \\
\hline \multirow[t]{4}{*}{$\begin{array}{l}\text { C-Reactive Protein } \\
(\mathrm{CRP})\end{array}$} & $\begin{array}{l}\text { Total Cases } \\
<6 \mathrm{mg} / \mathrm{L}\end{array}$ & $\begin{array}{c}64(63.3) \\
32(68)\end{array}$ & $\begin{array}{c}37(36.7) \\
15(32)\end{array}$ & $\begin{array}{c}101 \\
47\end{array}$ & $0.65^{* *}$ \\
\hline & 6-100 mg/L ( mildly raised) & $24(59)$ & $17(41)$ & 41 & \\
\hline & $>100 \mathrm{mg} / \mathrm{L}$ ( markedly raised) & $8(61)$ & $5(39)$ & 13 & \\
\hline & Total cases & $64(63.3)$ & $37(36.7)$ & 101 & \\
\hline
\end{tabular}

*Determined by Chi-square test, ${ }^{* *}$ determined by Fisher's Exact test.

The sensitivity, specificity, positive predictive value (PPV), negative predictive value (NPV) and diagnostic accuracy were calculated for each of three parameters using formulas mentioned in Table-II. LDH had highest sensitivity $(75 \%)$ with the positive predictive value (PPV) of $71.6 \%$ and diagnostic accuracy of $65.3 \%$ (Table-I). CRP showed poor diagnostic accuracy of $46.5 \%$ with sensitivity of only $50 \%$ and positive predictive value (PPV) of 59.3\% (Table-I). The chi-square test showed that LDH levels had significant difference $(p$ value $=0.02$ ) between the RT-PCR positive and negative groups (Table-II). The Fisher's exact test used for CRP and ferritin showed insignificant difference of their levels in RT-PCR positive and negative groups (TableII). The Receiver Operator Characteristic (ROC) graph was made to see the area under the curve (AUC) of the studied parameters to prognosticate the patients with positive RT-PCR for COVID-19. LDH (AUC $=0.65)$ and Ferritin (AUC=0.59) had the curve above the reference line. $C R P$ had $A U C=0.42$ only (Figure). The suggested the cut-off for LDH is $>490 \mathrm{U} / \mathrm{L}$ (sensitivity: $70.3 \%$ ) and for Ferritin is $>152 \mathrm{ng} / \mathrm{L}$ (sensitivity: $68.8 \%$ ).

\section{DISCUSSION}

Sensitivity and specificity are common terms used in the settings of validity of a laboratory test. Sensitivity denotes accuracy and the term specificity is used for reproducibility. A receiver operator characteristic (ROC) curve denotes the relation between clinical sensitivity and specificity for every possible cut-off. It is used to assess that how accurate the test (s) in question is as compared to the gold standard test for the diagnosis of a disease. An AUC of 0.5 suggests no discrimi-
Our results revealed $\mathrm{LDH} \quad(\mathrm{AUC}=0.65)$ and Ferritin (AUC $=0.59$ ) had the curve above the reference line indicating that they have better accuracy than CRP (AUC $=0.42$ ) in predicting the positive RT-PCR for COVID-19 (Figure). That indicated their predictive ability for detecting presence of COVID-19. CRP (AUC $=0.42$ ) proved it to be a poor predictor of the disease.

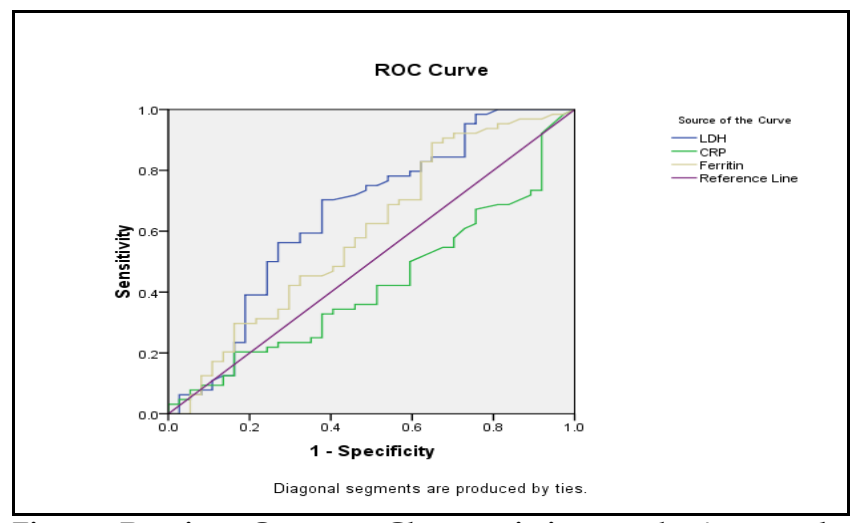

Figure: Receiver Operator Characteristic graph. Area under the curve (AUC) of $\mathrm{LDH}=0.65$, AUC of Ferritin $=0.59$ and AUC of $R P=0.42$.

Our findings in regard of CRP were in contrast to other studies. Only LDH levels had significant difference between RT-PCR positive and negative groups $(p=$ 0.02) (Table-II). It may be because the sample size was small and with increase in sample size, more differences that are prominent may be observed.

Chang et al, concluded that raised LDH is an independent risk factor for severity (HR: $2.73,95 \%$ CI: 1.25-5.97; $p=0.012$ ) and mortality (HR: $40.50,95 \% \mathrm{CI}$ : 3.65-449.28; $p=0.003$ ) of COVID-19.5 A study by Wang 
et al, supports our findings as they found that COVID19 of significant severity is associated with $\mathrm{LDH}>245$ $\mathrm{U} / \mathrm{L}(\mathrm{AUC}=0.82), \mathrm{CRP}>64.79$ (AUC=0.78) in addition to increased age, D-dimers, serum amyloid and decreased serum Albumin 9 . In COVID-19 lungs are mainly involved leading to hypoxia, thrombogenesis and inflammatory damage to the lung tissue. Raised LDH level indicates tissue destruction and is considered an important prognostic marker for lung injury. ${ }^{12} \mathrm{~A}$ very similar diagnostic accuracy study was conducted by Mardani et al, in Iran. They found that including the parameters like serum ALT, Neutrophils count, Urea, the CRP (AUC=0.870) and LDH (AUC=0.835) had a remarkable accuracy in predicting patients with positive RT-PCR for COVID-19.13 Most of the studies suggest that LDH is raised during SARS-CoV-2 infection and has good predictive ability for the presence of COVID19. Tsui and colleagues reported that on admission elevated level LDH and Neutrophil count were independent predictors of a poor clinical outcome. ${ }^{14}$ Our findings are in agreement that high LDH levels are associated with severe disease or higher viral load.11-13

Lui et al, from China, conducted a study in year 2020, including 12 patients showed that CRP levels negatively correlated with viral load. ${ }^{15}$ Among the biochemical indexes, the AUC on ROC curve for the infection and tissue damage indicators, Albumin, CRP, and LDH were 1, 0.938, and 0.844, respectively. These may also be potential predictors of disease severity. However, sample size of their study was too small to generalize the result. In contrast to their findings, our study showed that CRP is a poor predictor of the RTPCR positivity for COVID-19 and is poorly related to the disease. Another study done on 25 patients by Azzi et al showed no significant association between CRP and viral load in COVID-19 patients. ${ }^{12}$

In agreement to our findings, three large studies reported significant increase in serum ferritin along with other biochemical parameters in non-survivors compared to survivors. ${ }^{11,16,17}$ In a large multi-centered study many demographic and biochemical parameters were compared between survivors and non-survivors of COVID-19 using multivariable regression. ${ }^{11}$ Significant difference in the levels of Ferritin and LDH was observed between two groups. Feld et al, also studied ferritin as a predictor of mortality in 942 cases of COVID-19.18 Ferritin levels on admission and the maximum levels observed were poor predictors of death on ROC analysis, with AUC of 0.77 and 0.638 respectively.
We infer that a raised LDH (>490 U/L) and Ferritin (>152 ng/L) in symptomatic patients, suspected of COVID-19, should alert us. Such suspected patients should be immediately isolated and labeled as highrisk cases. With early identification, isolation and management we can control spread of disease in any population.

\section{LIMITATION OF STUDY}

Our study had few limitations as we studied only three biochemical parameters in symptomatic suspected COVID19 patients. We recommend that for better analysis, large scale study, including hematological, biochemical and other inflammatory markers, should be done.

\section{CONCLUSION}

We conclude that LDH and Ferritin have good accuracy and can be helpful in prognosticating the presence of COVID-19 disease in symptomatic suspected patients. On the other hand, CRP is a poor indicator/marker of positive RTPCR for COVID-19.

\section{Conflict of Interest: None.}

\section{Authors' Contribution}

TA: Design, collection and analysis of data, writing initial draft, AS: Revised and editing, NU: Data writing, revised and editing the data, QB: Data analysis, SS: Conceived and designed the study, AI: Revised and editing.

\section{REFERENCES}

1. World Health Organization. Public health surveillance for COVID-19: interim guidance, 16 December 2020. World Health Organization. 2020, Available at: https://apps.who.int/iris/ handle/10665/337897. License: CC BY-NC-SA 3.0 IGO. [Accessed on March 8, 2021]

2. McIntosh, MD K. UpToDate [Internet]. Uptodate.com. 2021 Available from: https://www.uptodate.com/contents/ coronavirus-disease-2019-covid-19-epidemiology-virology-andprevention [Accessed on March 10, 2021].

3. COVID-19 Health Advisory Platform by Ministry of National Health Services Regulations and Coordination [Internet]. Covid.gov.pk. 2021, [Internet] Available from: http://covid.gov. pk/guideline. [ Accessed on March 12, 2021]

4. Abid K, Bari YA, Younas M, Tahir Javaid S, Imran A. Progress of COVID-19 Epidemic in Pakistan. Asia Pac J Public Health 2020; 32(4): 154-156.

5. Li C, Ye J, Chen Q, Hu W, Wang L, Fan Y, et al. Elevated Lactate Dehydrogenase $(\mathrm{LDH})$ level as an independent risk factor for the severity and mortality of COVID-19. Aging (Albany NY).2020; 12(15): $15670-15681$.

6. Sethuraman N, Jeremiah SS, Ryo A. Interpreting Diagnostic Tests for SARS-CoV-2. JAMA 2020; 323(22): 2249-2251.

7. Ponti G, Maccaferri M, Ruini C, Tomasi A, Ozben T. Biomarkers associated with COVID-19 disease progression. Crit Rev Clin Lab Sci 2020; 57(6): 389-399.

8. Yuan J, Zou R, Zeng L, Kou S, Lan J, Li X, et al. The correlation between viral clearance and biochemical outcomes of 94 COVID19 infected discharged patients. Inflamm Res 2020; 69(6): 599-606.

9. Wang D, Li R, Wang J, Jiang Q, Gao C, Yang J, et al. Correlation analysis between disease severity and clinical and biochemical 


\section{Diagnostic Accuracy of Biochemical Parameters in COVID}

characteristics of 143 cases of COVID-19 in Wuhan, China: a descriptive study. BMC Infect Dis 2020; 20(1): 1-9.

10. Tan C, Huang Y, Shi F, Tan K, Ma Q, Chen Y, et al. C-reactive protein correlates with computed tomographic findings and predicts severe COVID-19 early. J Med Virol 2020; 92(7): 856-862.

11. Flahault A, Cadilhac M, Thomas G. Sample size calculation should be performed for design accuracy in diagnostic test studies. J Clin Epidemiol 2005; 58(8): 859-862.

12. Zhou F, Yu T, Du R, Fan G. Clinical course and risk factors for mortality of adult inpatients with COVID-19 in Wuhan, China: a retrospective cohort study. Lancet 2020; 395(10229): 1054-1062.

13. Rao SN, Manissero D, Steele VR, Pareja J. A narrative systematic review of the clinical utility of cycle threshold values in the context of COVID-19. Infect Dis Ther 2020; 9(3): 573-586.

14. Mardani R, Zali F, Gholami A, Mousavi Nasab SD, Kaghazian H, Kaviani M, et al. Laboratory parameters in detection of COVID19 patients with positive RT-PCR; a diagnostic accuracy study. Arch Acad Emerg Med 2020; 8(1): e43-e50.
15. Tsui PT, Kwok ML, Yuen H, Lai ST. Severe acute respiratory syndrome: clinical outcome and prognostic correlates. Emerg Infect Dis 2003; 9(9): 1064-1069.

16. Liu Y, Yang Y, Zhang C, Huang F, Wang F, Yuan J, et al. Clinical and biochemical indexes from 2019-nCoV infected patients linked to viral loads and lung injury. Sci China Life Sci 2020; 63(3): 364-374.

17. Yang $X, Y u$ Y, Xu J, Shu H, Xia Ja, Liu H, et al. Clinical course and outcomes of critically ill patients with SARS-CoV-2 pneumonia in Wuhan, China: a single-centered, retrospective, observational study. Lancet Respir Med 2020; 8(5): 475-481.

18. Ruan Q, Yang K, Wang W, Jiang L, Song J. Clinical predictors of mortality due to COVID-19 based on an analysis of data of 150 patients from Wuhan, China. Crit Care Med 2020; 46(5): 846-848.

19. Feld J, Tremblay D, Thibaud S, Kessler A, Naymagon L. Ferritin levels in patients with COVID-19: A poor predictor of mortality and hemophagocytic lymphohistiocytosis. Int J Lab Hematol 2020; 42(6): 773-779. 